\section{Tuberculin Anergy}

Transient depression of the tuberculin reaction is seen during the course of the acute exanthemata, particularly measles, scarlet fever, pertussis, influenza, typhus, and pneumonia. It has also been observed after vaccination against measles, poliomyelitis, and yellow fever, after ingestion of alcohol, exposure to sunlight or irradiation, and during hypnosis. ${ }^{1-3}$ A more permanent diminution or loss of the reaction may be seen in sarcoidosis, lymphoma, chronic leukaemia, amyloidosis, syphilis, hypothryoidism, and advanced carcinoma. ${ }^{1}{ }^{4}$ The administration of steroids either systemically or locally may render previously positive reactions negative. ${ }^{5-8}$

In patients with tuberculosis the tuberculin test is negative during the incubation period of the disease, within three to nine weeks of infection, while the cell-mediated delayed hypersensitivity is being built up. The reaction may become negative in patients severely ill with tuberculosis, but it becomes positive again if they recover on treatment. Less commonly the reaction is negative in apparently healthy people with active tuberculosis. ${ }^{1}{ }^{9} 10$ The real frequency of negative reactions in patients with active tuberculosis is somewhat obscured by the different doses of old tuberculin or purified protein derivative that have been employed for the test and also by the different criteria used in determining whether a reaction is positive or not. However, the larger the dose given the fewer patients have negative reactions. ${ }^{11}$

W. L. Howard and his colleagues ${ }^{12}$ have recently drawn attention to the loss of tuberculin sensitivity in patients with active tuberculosis. In a study of almost 10,000 patients seen over 30 years they found negative tuberculin reactions to $10 \mathrm{mg}$. of old tuberculin (1 mg. of old tuberculin is equivalent to $0.005 \mathrm{mg}$. of purified protein derivative) in $0.55 \%$, an incidence similar to that in several other series. ${ }^{1}{ }^{9} 11$ These patients were always critically ill and wasted, they had numerous acid-fast bacilli in their sputum, and their chest radiographs showed cavitation with additional evidence of recent spread of the disease. They also had high white blood counts with $90 \%$ or more polymorphonuclear leucocytes. The histology of areas in the lungs where recent spread had occurred showed that the tubercle bacilli had caused an exudative reaction without tissue necrosis, such as one might find in acute pneumococcal pneumonia.

Several mechanisms have been proposed to account for tuberculin anergy. As the tuberculin reaction can be abolished in tuberculous patients by injecting increasing doses of tuberculin ${ }^{7}$ it has been suggested that the anergy seen in patients severely ill may be due to desensitization by the tuberculoprotein released from their tubercle bacilli. This does not explain the negative reactions in those patients who remain well and whose reactions stay negative throughout their disease.

The loss of sensitivity that occurs in virus infections may be due to viral parasitism of the cells responsible for maintaining delayed hypersensitivity, but it has also been suggested that it is due to the increase in circulating steroids that accompanies an acute illness. ${ }^{13}$ There is some circumstantial evidence to support this view. Patients dying from carcinoma of the bronchus ${ }^{14}$ and other sites have raised levels of plasma cortisol, and the levels tend to become higher as death approaches. Such patients have diminished or absent reactions to tub erculin. Similarly patients critically ill from other diseases, ${ }^{15} 16$ including one with disseminated tuberculosis, ${ }^{17}$ have been shown to have abnormally high plasma cortisol levels. Furthermore steroids diminish delayed hypersensitivity reactions to a greater extent than humoral antibody reactions, a situation which exists in patients with advanced cancer. ${ }^{4}$ Cortisone reduces the size of the tuberculin reaction in tuberculous patients with known sensitivity, when given systemically ${ }^{18}$ or locally, and the inhibitory effect of cortisone on the reaction diminishes with diminishing tuberculin sensitivity. ${ }^{7}$ In contrast, in patients who have been desensitized to tuberculin and also in patients with sarcoidosis cortisone induces positive reactions in $70 \%$ and $50 \%$ respectively. ${ }^{?}$

In sarcoidosis it has also been shown that the anergic state is non-specific, for similar degrees of suppression are present for other antigens, but the ability to form humoral antibodies remains intact, ${ }^{19}$ while in lyphoma both delayed hypersensitivity and humoral antibody production are impaired..$^{20}$ Nowhere is there convincing evidence that specific tuberculin anergy exists. The evidence points more towards damage to or inhibition of the immune mechanisms that are concerned with delayed hypersensitivity in general rather than tuberculin in particular.

The mechanism of tuberculin non-reactivity in the patients who remain quite well in spite of active tuberculosis is even more obscure. Some have reacted to tuberculin but not purified protein derivative ${ }^{1}$ and some have "anticutins" in their serum, similar to those found in sarcoidosis, that inhibit the tuberculin reaction. ${ }^{9}$ Whether their anergy is specific or non-specific for tuberculin is not clear but it is a permanent phenomenon. Perhaps a few have an inherited or congenital inability to develop delayed hypersensitivity reactions, and studies of both humoral antibody production and delayed hypersensitivity to other antigens in such patients and their families might be rewarding.

1 Kent, D. C., and Schwartz, R., American Review of Respiratory Diseases, $1967,95,411$.

2 Brody, J. A., Overfield, T., and Hammes, L. M., New England Fournal of Medicine, 1964, 271, 1294.

s Black, S., Humphrey, J. H., and Niven, J. S. F., British Medical Fournal, $1963,1,1649$.

+ Ashikawa, K., Motoya, K., Sekiguchi, M., and Ishibashi, Y., Gann, 1967, $58,565$.

5 Salomon, H., and Angel, J. H., American Review of Respiratory Diseases, $1961,83,235$.

- Tsacalidis, D., Manios, S., Kanakoudi, F., and Charocopos, S., Helvetica Paediatrica Acta, 1969, 24, 323.

7 Citron, K. M., and Scadding, J. G., Quarterly Fournal of Medicine, 1957, 26, 277.

B Johnston, R. N., Ritchie, R. T., and Murray, I. H. F., British Medical fournal, 1963, 2, 720.

- Mascher, W., American Review of Tuberculosis, 1951, 63, 501.

10 Scadding, J. G., Tubercle, 1956, 37, 371.

11 Lester, C. F., and Atwell, R. L., American Review of Respiratory Diseases, $1958,78,399$.

12 Howard, W. L., Klopfenstein, M. D., Steininger, W. J., and Woodruff, C. E., Chest, 1970, 57, 530.

13 Starr, S., and Berkovich, S., New England Fournal of Medicine, 1964 $270,386$.

14 Werk, E. E., Sholiton, L. J., and Marnell, R. T., American Fournal of Medicine, 1963, 34, 192.

15 Nelson, D. H., Cooper, C. E., Gold, E. M., and Rutherford, E. R., fournal of Clinical Investigation, 1961, 40, 1067.

16 Nelson, D. H., Samuels, L. T., Willardson, D. G., and Tyler, F. H., fournal of Clinical Endocrinology, 1951, 11, 1021.

17 Perkoff, G. T., Sandberg, A. A., Nelson, D. H., and Tyler, F. H., Archives of Internal Medicine, 1954, 93, 1 .

18 Lovell, R. R. H., Goodman, H. C., Hudson, B., Armitage, P., and Pickering, G. W., Clinical Science, 1953, 12, 41.

1. Sones, M., and Israel, H. L., Annals of Internal Medicine, 1954, 40, 260.

20 Schier, W. W., New England fournal of Medicine, 1954, 250, 353. 